Kresna Social Science and Humanities Research

Proceedings of the International Conference On Ummah:

Digital Innovation, Humanities And Economy (ICU: DIHEc) 2020

https://doi.10.30874/ksshr.63

\title{
Innovation Techniques Analisys in Macroeconomy on Ratio of Financial Islamic Bank
}

\author{
Ahmad Subagyo \\ STIE GICI Depok \\ bagyo1972@gmail.com \\ $\underline{62-812-1312-2792}$ \\ Roberto Akyuwen \\ Pascasarjana MM Universitas Panca Sakti Tegal \\ Akyuwenroberto@gmail.com \\ Desmadi Saharuddin \\ Lecturer of Universitas Islam Negeri Syarif Hidayatullah Jakarta - Indonesia \\ Email: desmadi.saharuddin@ uinjkt.ac.id
}

\begin{abstract}
This study aims to investigate macro-economic variables on the financial ratios of Islamic banks in Indonesia, using simultaneous impulse response function (IRF) and forecast error variance decomposition (FEVD) analisys. The object in the sample research is one of the Islamic banks in Indonesia, namely the bank muamalah. The data used in this study consists of 4 macroeconomic variables of Indonesia and 4 variable ratio of Islamic banks in Indonesia. From the research that has been done macro economic variable response is still very volatile in the first month until month 10, positive and negative response (up and down) since the occurrence of shock or shock to the variable banking sector. Next, from the 9th to the 10th month the fluctuations begin to shrink meaning that the macroeconomic variables are no longer very volatile like the previous period. By using Impulse Response (ROA) in the results that in the first period of variable banking ROA ratio is strongly influenced by FDR shock (12.6\%) while the period of the period of shock ROA and other variables still not give influence.
\end{abstract}

Keywords: IRF, FEVD, Macroeconomics, financial performance of islamic bank JEL:

ISSN 2774-3918 (online), https://ksshr.kresnanusantara.co.id. Published by Kresna Nusantara Copyright @ Author(s). This is an open-access article distributed under the terms of the Creative Commons Attribution License (CC BY). To view a copy of this license, visit https://creativecommons.org/licenses/by/4.0/. 


\section{Kresna Social Science and Humanities Research}

Proceedings of the International Conference On Ummah:

Digital Innovation, Humanities And Economy (ICU: DIHEc) 2020

https://doi.10.30874/ksshr.63

\section{INTRODUCTION}

In a few decades, a discourse arose in the establishment of a sharia banking financial institution, which in value could make the State macro policy stable. In addition there are also some obstacles that will likely occur with the operation of Islamic banks in Indonesia. Indirectly again can bring up the discourse, what exactly can be influenced by the presence and emergence of Islamic banking in Indonesia. Quoted from a paper there are several possibilities that can occur or that can occur if the operation of Islamic banking in a country. This is of course related to the economic condition of the State, which may or may not be influenced by micro or macro with the emergence of sharia banking. If seen in general, the emergence of Islamic banking can be associated with economic conditions, such as growth rates or equity in terms of income and economy in Indonesia. The possibility of explaining why sharia banking needs to be developed and developed [1], the first is definitely related to economic stability, especially related to sharia banking transactions. By using the system of sharia and Islamic law is in balance value in the economy will be able to provide a macro impact, in because most of the population in Indonesia islam religion. Selanjutanya certainly associated with a larger market share, which may be achieved by sharia banking. In order to maintain the stability that will be developed in sharia banking in Indonesia, market share capital is considered sufficient to see the significant effect that will occur on the inflation rate and the scarcity of goods .

While the last is the impact of sharia banking operations on equity. Islamic banking currently operates using a profit-sharing system. "A fair and equitable sharing system on the funding side as well as on the financing side will have an equity impact,". With the addition of basic philosophy that carried Islamic banking in the form of Natural Uncertain Contract then the profit -sharing system will apply the principle of little results or large results will be shared fairly. "Thus is the form of efficiency of sharia banking, and the key to success is the low Non Performing Financing,". With the emergence of this impact, the authors want to examine the extent to which the influence of macroeconomic variables that exist in Indonesia against Islamic banks, in review of the empirical study of a study.

\section{LITERATURE REVIEW}

In the study conducted [2 \& 3] discussed also about economic stabilization and restructuring process in the banking sector and privatization process which is considered more conducive in the transition period. The occurrence of the global financial crisis that could make the banking sector crisis, make the example and the impetus to be able to make a banking sector in the economy become stronger, both legally and economically. Some of the ratios found in the banking industry, such as capital adequacy ratio, credit ratio and minimum mandatory gro ratio, make industry in the banking sector can be systemically risky. The existence of good macroeconomic stability can have an indirect impact on financial stability of banks, such as the NPL ratio. In their study, the use of macroeconomic variables and banking ratios, in the basis of data and analysis in assessing the economic growth and stability of banks in the country in doing research that is Poland and Hungary. Given the positive economic recovery is considered to be able to spur performance in

ISSN 2774-3918 (online), https://ksshr.kresnanusantara.co.id. Published by Kresna Nusantara

Copyright (c) Author(s). This is an open-access article distributed under the terms of the Creative Commons Attribution License (CC BY). To view a copy of this license, visit https://creativecommons.org/licenses/by/4.0/. 


\section{Kresna Social Science and Humanities Research}

Proceedings of the International Conference On Ummah:

Digital Innovation, Humanities And Economy (ICU: DIHEc) 2020

https://doi.10.30874/ksshr.63

the banking sector. They considered that the crisis in Hungary or more known as the depreciation of Forint and the crisis in Poland, known as Polish Zloty, contributed to the economies of both countries, the impact of higher lending market share, and more in the dominated by the eyes foreign money. The banking crisis in Hungary caused the NPL ratio to rise, while in this case the local government did not provide protection to the banking world for the guarantee of this NPL increase, so that many industries outside the banking sector went bankrupt.

In other studies [4,5,6 \& 7], testing almost the same thing, namely macroeconomics and banking ratios. Macroeconomic variables that are used as an analytical tool not far from GDP, Inflation and the amount of government debt ratio. As for the performance of banking variables that are used in their study can not be separated from the ratio of NPL, CAR and ROA. Using wald test and causality, in their study found a short-term causal relationship between the variables in the study, but before the causality test was done, in their study using stationary test data, to see the level of kestasioneritas of data that will be used in research. In addition to the data they tested, stationary is also normally distributed, but the data must first be changed into logarithmic form. The end result of their NPL studies and non-performing loans ratio is related to growth rates in the countries where they conduct research.

In the study [8,9] justify the results described in the studies $[4,5,6 \& 7]$, however the analysis of the data in use is slightly different and more complex. VAR modelers and cointegration can not be separated from the studies [8,9], but still the VECM modeling can explain the results of their studies. But the data test they do not be separated from autocorrelation, heteroscedasticity test, causality. To explain the effect of macroeconomic variables on the variables of the banking sector using the VAR test, it can be seen how the results of a commercial lending rate, GDP ratio and debt ratio, simultaneously to the ratio of problem banking credit. The overall conclusion explains that there is a long-term and short-term relationship between the variables conducted in the study. And in long-term balance, the error of the model is corrected by using the VECM test. So there is a long term causal relationship between macroeconomic variables and banking variables that they use as research data. However, in the long term, unintentionally the ratio of nonperforming loans affects the value of commercial lending rates.

\section{RESEARCH METHOD}

For this research is done by the authors in February 2018. This study consists of 4 economic variables and 4 variable performance of sharia banks. The sharia bank in which the sample is made is the Indonesian muamalat bank. In this research to make some variables, where the variables include BI RATE, BOPO, index rate, IPI bank, consumer price index, FDR, inflation Indonesia and ROA. For the report of bank financial ratios used in accordance with the financial statements of muamalat banks for the year ending 2010 to 2016.

\section{RESULT AND DISCUSSION}

ISSN 2774-3918 (online), https://ksshr.kresnanusantara.co.id. Published by Kresna Nusantara

Copyright (c) Author(s). This is an open-access article distributed under the terms of the Creative Commons Attribution License (CC BY). To view a copy of this license, visit https://creativecommons.org/licenses/by/4.0/. 


\section{Kresna Social Science and Humanities Research}

Proceedings of the International Conference On Ummah:

Digital Innovation, Humanities And Economy (ICU: DIHEc) 2020

https://doi.10.30874/ksshr.63

\section{Impulse response function (IRF)}

The method of impulse response function analysis is a tool used to detect dynamic relationships among variables in a system of equations. In other words a shock to a particular variable will directly affect the variable it self, then the effect will be spread to all other endogenous variables through dynamic structure or lag structure VAR used in the equation [10]. Therefore this analytical tool can be used to perform dynamic simulation for future forecasting. Here are the results of the forecast for the variables of this study: [presented in table 1].

Table 1 : Result for impulse response function (IRF)

\begin{tabular}{|c|c|c|c|c|c|c|c|c|}
\hline \multicolumn{9}{|c|}{ Response of BI_RATE: } \\
\hline Period & BI_RATE & BOPO & FDR & IHK & IPI & KURS & INF & ROA \\
\hline \multirow[t]{2}{*}{1} & 0.103913 & 0.000000 & 0.000000 & 0.000000 & 0.000000 & 0.000000 & 0.000000 & 0.000000 \\
\hline & $(0.00811)$ & $(0.00000)$ & $(0.00000)$ & $(0.00000)$ & $(0.00000)$ & $(0.00000)$ & $(0.00000)$ & $(0.00000)$ \\
\hline \multirow[t]{2}{*}{2} & 0.149559 & -0.013932 & 0.020670 & -0.019407 & -0.016400 & 0.004855 & 0.002648 & 0.000627 \\
\hline & $(0.01666)$ & $(0.01258)$ & $(0.01183)$ & $(0.01205)$ & $(0.01189)$ & $(0.01155)$ & $(0.01191)$ & $(0.01227)$ \\
\hline \multirow[t]{2}{*}{3} & 0.162561 & -0.014075 & 0.033370 & -0.017901 & -0.011286 & 0.007980 & 0.017965 & -0.012795 \\
\hline & $(0.02528)$ & $(0.02153)$ & $(0.01990)$ & $(0.01913)$ & \begin{tabular}{|l|}
$(0.02037)$ \\
\end{tabular} & $(0.01677)$ & $\begin{array}{l}(0.02059) \\
\end{array}$ & $(0.02085)$ \\
\hline \multirow[t]{2}{*}{4} & 0.168191 & -0.001593 & 0.033654 & -0.009259 & -0.011877 & 0.007791 & 0.023890 & -0.019125 \\
\hline & $(0.03151)$ & $(0.02680)$ & $(0.02646)$ & $(0.02393)$ & $(0.02677)$ & $(0.01981)$ & $(0.02621)$ & $(0.02808)$ \\
\hline \multirow[t]{2}{*}{5} & 0.161182 & 0.002367 & 0.034891 & -0.002545 & -0.016456 & 0.007245 & 0.027282 & -0.021281 \\
\hline & \begin{tabular}{|l|}
$(0.03633)$ \\
\end{tabular} & $(0.02930)$ & $(0.03135)$ & \begin{tabular}{|l}
$(0.02825)$ \\
\end{tabular} & \begin{tabular}{|l|l}
$(0.03040)$ \\
\end{tabular} & $(0.02237)$ & \begin{tabular}{|l|}
$(0.03119)$ \\
\end{tabular} & \begin{tabular}{|l}
$(0.03271)$ \\
\end{tabular} \\
\hline \multirow[t]{2}{*}{6} & 0.145827 & 0.001104 & 0.032839 & 0.004523 & -0.016965 & 0.003799 & 0.029842 & -0.026326 \\
\hline & \begin{tabular}{|l|}
$(0.03990)$ \\
\end{tabular} & $(0.02967)$ & $(0.03493)$ & \begin{tabular}{|l|}
$(0.03119)$ \\
\end{tabular} & $(0.03234)$ & $(0.02396)$ & \begin{tabular}{|l}
$(0.03485)$ \\
\end{tabular} & $(0.03465)$ \\
\hline \multirow[t]{2}{*}{7} & 0.129440 & 0.001299 & 0.027445 & 0.010514 & -0.016065 & -0.001861 & 0.029355 & -0.029124 \\
\hline & \begin{tabular}{|l|}
$(0.04245)$ \\
\end{tabular} & $(0.02848)$ & $(0.03731)$ & \begin{tabular}{|l|}
$(0.03257)$ \\
\end{tabular} & \begin{tabular}{|l|}
$(0.03276)$ \\
\end{tabular} & $(0.02472)$ & \begin{tabular}{|l|}
$(0.03656)$ \\
\end{tabular} & \begin{tabular}{|l|}
$(0.03477)$ \\
\end{tabular} \\
\hline \multirow[t]{2}{*}{8} & 0.112762 & 0.000185 & 0.021627 & 0.014343 & -0.015334 & -0.007133 & 0.027585 & -0.029596 \\
\hline & \begin{tabular}{|l|}
$(0.04445)$ \\
\end{tabular} & $(0.02679)$ & $(0.03864)$ & \begin{tabular}{|l}
$(0.03274)$ \\
\end{tabular} & $(0.03193)$ & $(0.02504)$ & \begin{tabular}{|l|}
$(0.03665)$ \\
\end{tabular} & \begin{tabular}{|l|}
$(0.03379)$ \\
\end{tabular} \\
\hline \multirow[t]{2}{*}{9} & 0.095932 & -0.001701 & 0.015688 & \begin{tabular}{|l|}
0.017246 \\
\end{tabular} & -0.013601 & \begin{tabular}{|l|}
-0.012294 \\
\end{tabular} & 0.025456 & \begin{tabular}{|l|}
-0.029763 \\
\end{tabular} \\
\hline & \begin{tabular}{|l|}
$(0.04605)$ \\
\end{tabular} & \begin{tabular}{|l}
$(0.02516)$ \\
\end{tabular} & $(0.03910)$ & \begin{tabular}{|l}
$(0.03195)$ \\
\end{tabular} & $(0.03034)$ & $(0.02493)$ & \begin{tabular}{|l|}
$(0.03549)$ \\
\end{tabular} & \begin{tabular}{|l}
$(0.03229)$ \\
\end{tabular} \\
\hline \multirow[t]{2}{*}{10} & 0.080211 & -0.003105 & 0.009692 & \begin{tabular}{|l|}
0.019489 \\
\end{tabular} & -0.011569 & -0.017187 & 0.022595 & -0.029408 \\
\hline & $(0.04727)$ & $(0.02362)$ & $(0.03874)$ & $(0.03048)$ & $(0.02821)$ & $(0.02444)$ & $(0.03345)$ & $(0.03059)$ \\
\hline \multicolumn{9}{|c|}{ Response of BOPO: } \\
\hline Period & BI_RATE & BOPO & FDR & IHK & IPI & KURS & INF & ROA \\
\hline \multirow[t]{2}{*}{1} & 0.509448 & 2.861332 & 0.000000 & 0.000000 & 0.000000 & 0.000000 & 0.000000 & 0.000000 \\
\hline & $(0.31848)$ & $(0.22343)$ & $(0.00000)$ & $(0.00000)$ & $(0.00000)$ & $(0.00000)$ & $(0.00000)$ & $(0.00000)$ \\
\hline \multirow[t]{2}{*}{2} & 0.310554 & \begin{tabular}{|l|}
0.081210 \\
\end{tabular} & \begin{tabular}{|l|}
-0.075540 \\
\end{tabular} & -0.327741 & \begin{tabular}{|l|}
-0.573169 \\
\end{tabular} & \begin{tabular}{|l|}
0.657830 \\
\end{tabular} & \begin{tabular}{|l|}
0.150632 \\
\end{tabular} & -0.428434 \\
\hline & $(0.33468)$ & $(0.35448)$ & $(0.33783)$ & $(0.34831)$ & $(0.34477)$ & $(0.33076)$ & $\begin{array}{l}(0.33669) \\
\end{array}$ & $(0.34480)$ \\
\hline \multirow[t]{2}{*}{3} & 0.044090 & -0.280221 & -0.150271 & -0.150034 & -0.011805 & -0.247662 & 0.092511 & -0.663969 \\
\hline & \begin{tabular}{|l}
$(0.26899)$ \\
\end{tabular} & $(0.35039)$ & $(0.29547)$ & $(0.25319)$ & $(0.33375)$ & $(0.21832)$ & $(0.26354)$ & $(0.31823)$ \\
\hline \multirow[t]{2}{*}{4} & 0.527739 & \begin{tabular}{|l|}
-0.129199 \\
\end{tabular} & -0.194403 & -0.313769 & -0.200887 & -0.229564 & -0.080628 & -0.245706 \\
\hline & $(0.21602)$ & $(0.22636)$ & $(0.21730)$ & $(0.20463)$ & $(0.21046)$ & $(0.17072)$ & $(0.22933)$ & $(0.24386)$ \\
\hline \multirow[t]{2}{*}{5} & 0.577440 & -0.195015 & -0.014125 & -0.331302 & -0.147466 & -0.100355 & 0.076078 & -0.045409 \\
\hline & $(0.21065)$ & $\begin{array}{l}(0.17740) \\
\end{array}$ & $(0.18317)$ & $(0.15430)$ & $(0.16248)$ & $(0.12715)$ & $(0.18347)$ & $(0.17489)$ \\
\hline \multirow[t]{2}{*}{6} & 0.591303 & -0.070608 & 0.013893 & -0.203736 & -0.071087 & -0.060000 & 0.140751 & -0.069965 \\
\hline & $(0.20187)$ & $(0.15444)$ & \begin{tabular}{|l|l}
$(0.17211)$ \\
\end{tabular} & $(0.14491)$ & $(0.15140)$ & $(0.12002)$ & $(0.16164)$ & $(0.16655)$ \\
\hline
\end{tabular}




\section{Kresna Social Science and Humanities Research}

Proceedings of the International Conference On Ummah:

Digital Innovation, Humanities And Economy (ICU: DIHEc) 2020 https://doi.10.30874/ksshr.63

\begin{tabular}{|c|c|c|c|c|c|c|c|c|}
\hline \multirow[t]{2}{*}{7} & 0.588758 & 0.042748 & 0.063245 & -0.117070 & -0.099424 & -0.016711 & 0.120008 & -0.041702 \\
\hline & $(0.20055)$ & (0.13506) & $(0.16267)$ & $(0.13669)$ & $(0.13984)$ & \begin{tabular}{|l}
$(0.10783)$ \\
\end{tabular} & \begin{tabular}{|l}
$(0.15131)$ \\
\end{tabular} & \begin{tabular}{|l}
$(0.15403)$ \\
\end{tabular} \\
\hline \multirow[t]{2}{*}{8} & 0.512650 & 0.014812 & 0.105550 & -0.057838 & -0.109568 & 0.019112 & 0.115949 & -0.066813 \\
\hline & $(0.20183)$ & $(0.12647)$ & $(0.16146)$ & $(0.13490)$ & $(0.13292)$ & \begin{tabular}{|l}
$(0.10737)$ \\
\end{tabular} & \begin{tabular}{|l}
$(0.14909)$ \\
\end{tabular} & $(0.14776)$ \\
\hline \multirow[t]{2}{*}{9} & 0.436978 & 0.006426 & 0.097366 & -0.005916 & -0.080671 & 0.005194 & \begin{tabular}{|l|l|}
0.103929 \\
\end{tabular} & -0.097386 \\
\hline & $(0.20387)$ & $(0.11545)$ & $(0.16160)$ & $(0.13142)$ & $(0.12748)$ & $(0.10512)$ & $(0.14674)$ & $(0.14034)$ \\
\hline \multirow[t]{2}{*}{10} & 0.385937 & 0.008464 & 0.076115 & 0.016518 & -0.068612 & -0.010129 & 0.085856 & -0.093493 \\
\hline & $(0.20426)$ & $(0.10362)$ & $(0.15891)$ & $(0.12574)$ & $(0.11885)$ & $(0.10155)$ & \begin{tabular}{|l}
$(0.13966)$ \\
\end{tabular} & $(0.13192)$ \\
\hline \multicolumn{9}{|c|}{ Response of FDR: } \\
\hline Period & BI_RATE & BOPO & FDR & IHK & IPI & KURS & INF & ROA \\
\hline \multirow[t]{2}{*}{1} & -0.699188 & -0.383173 & 2.646900 & 0.000000 & 0.000000 & 0.000000 & 0.000000 & 0.000000 \\
\hline & $(0.30035)$ & $(0.29383)$ & $(0.20669)$ & $(0.00000)$ & $(0.00000)$ & $(0.00000)$ & $(0.00000)$ & $(0.00000)$ \\
\hline \multirow[t]{2}{*}{2} & -0.253981 & -0.613171 & 1.450798 & 0.416540 & 0.140636 & 0.246225 & -0.121192 & -0.288299 \\
\hline & $(0.35177)$ & $(0.36602)$ & $(0.32937)$ & $(0.31894)$ & \begin{tabular}{|l}
$(0.31738)$ \\
\end{tabular} & \begin{tabular}{|l}
$(0.30964)$ \\
\end{tabular} & \begin{tabular}{|l}
$(0.31860)$ \\
\end{tabular} & $(0.32719)$ \\
\hline \multirow[t]{2}{*}{3} & -0.113836 & 0.474728 & 0.921636 & 0.340492 & 0.287739 & -0.185958 & -0.184815 & 0.636365 \\
\hline & $(0.35990)$ & $(0.38371)$ & $(0.33251)$ & $(0.28699)$ & $(0.34877)$ & $(0.22906)$ & $(0.31500)$ & $(0.34183)$ \\
\hline \multirow[t]{2}{*}{4} & -0.224756 & 0.365350 & 0.716962 & 0.107947 & 0.067954 & 0.023205 & 0.061731 & 0.690887 \\
\hline & $(0.32452)$ & $(0.30959)$ & $(0.32103)$ & $(0.28374)$ & $(0.31282)$ & $(0.23128)$ & \begin{tabular}{|l}
$(0.31008)$ \\
\end{tabular} & $(0.34687)$ \\
\hline \multirow[t]{2}{*}{5} & -0.484809 & 0.374540 & 0.540087 & 0.198787 & 0.180186 & -0.129223 & 0.192595 & 0.383953 \\
\hline & \begin{tabular}{|l}
$(0.32477)$ \\
\end{tabular} & $(0.25013)$ & $(0.28607)$ & $(0.25786)$ & \begin{tabular}{|l}
$(0.24943)$ \\
\end{tabular} & \begin{tabular}{|l}
$(0.19031)$ \\
\end{tabular} & \begin{tabular}{|l}
$(0.29870)$ \\
\end{tabular} & $(0.28002)$ \\
\hline \multirow[t]{2}{*}{6} & -0.516285 & 0.367123 & 0.363061 & 0.213000 & 0.063036 & -0.220143 & 0.075597 & 0.251342 \\
\hline & $(0.31956)$ & $(0.21336)$ & $(0.27851)$ & $(0.23626)$ & $(0.23264)$ & \begin{tabular}{|l}
$(0.18190)$ \\
\end{tabular} & $(0.27145)$ & $(0.24829)$ \\
\hline \multirow[t]{2}{*}{7} & -0.590543 & 0.200222 & 0.282283 & 0.177444 & 0.012609 & -0.277477 & 0.010030 & 0.187053 \\
\hline & $(0.32024)$ & $(0.18496)$ & $(0.26903)$ & $(0.22198)$ & $(0.21311)$ & $(0.17051)$ & $(0.24797)$ & $(0.22646)$ \\
\hline \multirow[t]{2}{*}{8} & -0.622568 & 0.097575 & 0.162949 & 0.159818 & 0.045832 & -0.345203 & -0.029022 & 0.121622 \\
\hline & $(0.32429)$ & $(0.17269)$ & $(0.25982)$ & $(0.20755)$ & $(0.19491)$ & $(0.16589)$ & $(0.22713)$ & $(0.20941)$ \\
\hline \multirow[t]{2}{*}{9} & -0.596735 & 0.074534 & 0.060643 & 0.134955 & 0.063590 & -0.390012 & -0.067140 & 0.115750 \\
\hline & $(0.32934)$ & $(0.16740)$ & $(0.25397)$ & $(0.19730)$ & $(0.18451)$ & \begin{tabular}{|l}
$(0.16259)$ \\
\end{tabular} & $(0.21201)$ & $(0.20057)$ \\
\hline \multirow[t]{2}{*}{10} & -0.568580 & 0.039563 & -0.001223 & 0.111933 & 0.067057 & -0.401590 & -0.083539 & 0.107359 \\
\hline & $(0.33355)$ & $(0.16261)$ & $(0.24657)$ & $(0.18838)$ & $(0.17598)$ & \begin{tabular}{|l}
$(0.15921)$ \\
\end{tabular} & \begin{tabular}{|l}
$(0.19997)$ \\
\end{tabular} & $(0.19489)$ \\
\hline \multicolumn{9}{|c|}{ Response of IHK: } \\
\hline Period & BI_RATE & BOPO & FDR & IHK & IPI & KURS & INF & ROA \\
\hline \multirow[t]{2}{*}{1} & -0.032706 & -0.008677 & 0.017618 & 0.190493 & 0.000000 & 0.000000 & 0.000000 & 0.000000 \\
\hline & $(0.02130)$ & $(0.02114)$ & $(0.02108)$ & $(0.01487)$ & $(0.00000)$ & \begin{tabular}{|l}
$(0.00000)$ \\
\end{tabular} & \begin{tabular}{|l|}
$(0.00000)$ \\
\end{tabular} & $(0.00000)$ \\
\hline \multirow[t]{2}{*}{2} & -0.041712 & -0.010894 & -0.026944 & 0.153615 & -0.006453 & -0.018951 & -0.053372 & -0.015208 \\
\hline & $(0.02810)$ & $\begin{array}{l}(0.02902) \\
\end{array}$ & $(0.02803)$ & \begin{tabular}{|l}
$(0.02594)$ \\
\end{tabular} & $(0.02301)$ & \begin{tabular}{|l}
$(0.02248)$ \\
\end{tabular} & \begin{tabular}{|l}
$(0.02272)$ \\
\end{tabular} & \begin{tabular}{|l}
$(0.02297)$ \\
\end{tabular} \\
\hline \multirow[t]{2}{*}{3} & -0.057342 & -0.032 & -0.044215 & 0.103739 & 0.027842 & \begin{tabular}{|l|}
-0.027739 \\
\end{tabular} & -0.040745 & -0.028545 \\
\hline & \begin{tabular}{|l}
$(0.03189)$ \\
\end{tabular} & 0.032 & $(0.02935)$ & $(0.02$ & \begin{tabular}{|l}
$(0.02836)$ \\
\end{tabular} & \begin{tabular}{|l}
$(0.02059)$ \\
\end{tabular} & \begin{tabular}{|l|}
$(0.02674)$ \\
\end{tabular} & \begin{tabular}{|l}
$(0.02799)$ \\
\end{tabular} \\
\hline \multirow[t]{2}{*}{4} & -0.046602 & -0.036370 & -0.069068 & 0.075217 & 0.041560 & -0.041261 & -0.030708 & -0.042766 \\
\hline & $(0.03319)$ & $(0.03103)$ & $(0.03125)$ & $(0.027$ & $(0.02955)$ & \begin{tabular}{|l}
$(0.02079)$ \\
\end{tabular} & $(0.02841)$ & $(0.03130)$ \\
\hline 5 & -0.031195 & -0.031389 & -0.070655 & 0.053697 & \begin{tabular}{|l|l|}
0.032579 \\
\end{tabular} & -0.049139 & -0.027107 & -0.036992 \\
\hline & $(0.03475)$ & $(0.02869)$ & $(0.03160)$ & $(0.02826)$ & $(0.02833)$ & $(0.02113)$ & $(0.03071)$ & $(0.03073)$ \\
\hline 6 & -0.024338 & -0.036500 & -0.063060 & 0.040949 & 0.022898 & -0.047831 & -0.020980 & -0.036090 \\
\hline & \begin{tabular}{|l}
$(0.03568)$ \\
\end{tabular} & $(0.02653)$ & \begin{tabular}{|l}
$(0.03178)$ \\
\end{tabular} & \begin{tabular}{|l|}
$(0.02776)$ \\
\end{tabular} & \begin{tabular}{|l|}
$(0.02750)$ \\
\end{tabular} & \begin{tabular}{|l|}
$(0.02072)$ \\
\end{tabular} & \begin{tabular}{|l|}
$(0.03084)$ \\
\end{tabular} & \begin{tabular}{|l}
$(0.02914)$ \\
\end{tabular} \\
\hline 7 & -0.020715 & -0.034234 & -0.057979 & 0.036397 & 0.019618 & -0.048122 & -0.017619 & -0.036154 \\
\hline & $(0.03648)$ & $(0.02416)$ & $(0.03179)$ & $(0.02695)$ & $(0.02629)$ & $(0.02036)$ & $(0.02972)$ & \begin{tabular}{|l}
$(0.02749)$ \\
\end{tabular} \\
\hline 8 & -0.017174 & -0.029934 & -0.053935 & 0.032670 & 0.015534 & -0.046503 & -0.016972 & -0.032788 \\
\hline & \begin{tabular}{|l|}
$(0.03739)$ \\
\end{tabular} & $(0.02246)$ & $(0.03155)$ & \begin{tabular}{|l}
$(0.02598)$ \\
\end{tabular} & $(0.02484)$ & \begin{tabular}{|l}
$(0.02016)$ \\
\end{tabular} & $(0.02832)$ & \begin{tabular}{|l}
$(0.02592)$ \\
\end{tabular} \\
\hline 9 & -0.016354 & -0.028051 & -0.049745 & 0.029541 & 0.013188 & -0.044271 & -0.015652 & -0.030085 \\
\hline & \begin{tabular}{|l}
$(0.03819)$ \\
\end{tabular} & $(0.02119)$ & $(0.03127)$ & $(0.02494)$ & \begin{tabular}{|l|}
$(0.02354)$ \\
\end{tabular} & \begin{tabular}{|l}
$(0.01985)$ \\
\end{tabular} & \begin{tabular}{|l|}
$(0.02690)$ \\
\end{tabular} & 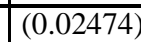 \\
\hline
\end{tabular}

ISSN 2774-3918 (online), https://ksshr.kresnanusantara.co.id. Published by Kresna Nusantara

Copyright (c) Author(s). This is an open-access article distributed under the terms of the Creative Commons Attribution License (CC BY). To view a copy of this license, visit https://creativecommons.org/licenses/by/4.0/. 


\section{Kresna Social Science and Humanities Research}

Proceedings of the International Conference On Ummah:

Digital Innovation, Humanities And Economy (ICU: DIHEc) 2020 https://doi.10.30874/ksshr.63

\begin{tabular}{|c|c|c|c|c|c|c|c|c|}
\hline 10 & -0.016131 & -0.025959 & -0.046758 & 0.027372 & 0.012277 & -0.042743 & -0.014570 & -0.028419 \\
\hline & $(0.03892)$ & $(0.02017)$ & $(0.03085)$ & \begin{tabular}{|l}
$(0.02392)$ \\
\end{tabular} & $(0.02222)$ & \begin{tabular}{|l|}
$(0.01954)$ \\
\end{tabular} & \begin{tabular}{|l}
$(0.02549)$ \\
\end{tabular} & $(0.02379)$ \\
\hline \multicolumn{9}{|c|}{ Response of IND_PROD_INDEX: } \\
\hline Period & BI_RATE & BOPO & FDR & IHK & IPI & KURS & INF & ROA \\
\hline \multirow[t]{2}{*}{1} & -0.474204 & -0.525880 & -0.232385 & -0.419789 & \begin{tabular}{|l|}
2.865757 \\
\end{tabular} & \begin{tabular}{|l|}
0.000000 \\
\end{tabular} & \begin{tabular}{|l|l|}
0.000000 \\
\end{tabular} & 0.000000 \\
\hline & \begin{tabular}{|l}
$(0.32818)$ \\
\end{tabular} & \begin{tabular}{|l}
$(0.32349)$ \\
\end{tabular} & \begin{tabular}{|l}
$(0.32036)$ \\
\end{tabular} & \begin{tabular}{|l|}
$(0.31816)$ \\
\end{tabular} & \begin{tabular}{|l|}
$(0.22378)$ \\
\end{tabular} & \begin{tabular}{|l|}
$(0.00000)$ \\
\end{tabular} & \begin{tabular}{|l}
$(0.00000)$ \\
\end{tabular} & \begin{tabular}{|l}
$(0.00000)$ \\
\end{tabular} \\
\hline \multirow[t]{2}{*}{2} & 0.164022 & 0.004922 & -0.162414 & -0.120689 & 0.908223 & \begin{tabular}{|l|}
-0.583596 \\
\end{tabular} & -0.804553 & -0.118814 \\
\hline & $(0.35621)$ & $(0.37662)$ & $(0.35993)$ & $(0.37100)$ & $(0.36434)$ & $(0.34718)$ & $(0.34877)$ & $(0.35326)$ \\
\hline \multirow[t]{2}{*}{3} & -0.066093 & -0.225579 & 0.513441 & -0.043773 & 0.206911 & -0.410596 & -0.562413 & 0.171936 \\
\hline & \begin{tabular}{|l|}
$(0.32030)$ \\
\end{tabular} & \begin{tabular}{|l|}
$(0.37463)$ \\
\end{tabular} & \begin{tabular}{|l|}
$(0.32069)$ \\
\end{tabular} & $(0.27514)$ & \begin{tabular}{|l|}
$(0.35253)$ \\
\end{tabular} & \begin{tabular}{|l|}
$(0.22326)$ \\
\end{tabular} & \begin{tabular}{|l}
$(0.29217)$ \\
\end{tabular} & \begin{tabular}{|l}
$(0.33669)$ \\
\end{tabular} \\
\hline \multirow[t]{2}{*}{4} & -0.261571 & -0.302419 & \begin{tabular}{|l|}
0.255813 \\
\end{tabular} & 0.216978 & \begin{tabular}{|l|}
0.323098 \\
\end{tabular} & \begin{tabular}{|l|}
-0.435746 \\
\end{tabular} & -0.270675 & -0.082170 \\
\hline & \begin{tabular}{|l}
$(0.27167)$ \\
\end{tabular} & \begin{tabular}{|l|}
$(0.26197)$ \\
\end{tabular} & \begin{tabular}{|l|}
$(0.27180)$ \\
\end{tabular} & \begin{tabular}{|l|}
$(0.24709)$ \\
\end{tabular} & \begin{tabular}{|l|}
$(0.26263)$ \\
\end{tabular} & \begin{tabular}{|l|}
$(0.19294)$ \\
\end{tabular} & \begin{tabular}{|l|}
$(0.26918)$ \\
\end{tabular} & \begin{tabular}{|l}
$(0.29783)$ \\
\end{tabular} \\
\hline \multirow[t]{2}{*}{5} & -0.210034 & 0.075771 & -0.002186 & 0.287910 & 0.293791 & -0.588298 & -0.247041 & 0.043679 \\
\hline & \begin{tabular}{|l}
$(0.26928)$ \\
\end{tabular} & \begin{tabular}{|l}
$(0.20817)$ \\
\end{tabular} & \begin{tabular}{|l|}
$(0.24156)$ \\
\end{tabular} & \begin{tabular}{|l|}
$(0.21536)$ \\
\end{tabular} & \begin{tabular}{|l|}
$(0.20863)$ \\
\end{tabular} & \begin{tabular}{|l|}
$(0.15866)$ \\
\end{tabular} & \begin{tabular}{|l|}
$(0.23978)$ \\
\end{tabular} & \begin{tabular}{|l}
$(0.22237)$ \\
\end{tabular} \\
\hline \multirow[t]{2}{*}{6} & -0.246290 & 0.010195 & -0.049041 & 0.231088 & 0.147359 & -0.549738 & -0.166977 & 0.060193 \\
\hline & $(0.26214)$ & $(0.17945)$ & $(0.23089)$ & $(0.19801)$ & $(0.19298)$ & $(0.15488)$ & $(0.21436)$ & $(0.19690)$ \\
\hline \multirow[t]{2}{*}{7} & -0.336387 & -0.065918 & -0.105981 & 0.263334 & 0.159004 & -0.602568 & -0.100082 & -0.070205 \\
\hline & $(0.26676)$ & $(0.16347)$ & \begin{tabular}{|l}
$(0.22475)$ \\
\end{tabular} & \begin{tabular}{|l}
$(0.18722)$ \\
\end{tabular} & $(0.18167)$ & \begin{tabular}{|l|}
$(0.14300)$ \\
\end{tabular} & $(0.19467)$ & (0.17862) \\
\hline \multirow[t]{2}{*}{8} & -0.332778 & -0.063434 & -0.181452 & 0.278726 & 0.130934 & -0.650314 & -0.121753 & -0.117646 \\
\hline & \begin{tabular}{|l|}
$(0.27708)$ \\
\end{tabular} & \begin{tabular}{|l}
$(0.16723)$ \\
\end{tabular} & \begin{tabular}{|l|}
$(0.22501)$ \\
\end{tabular} & \begin{tabular}{|l|}
$(0.18442)$ \\
\end{tabular} & \begin{tabular}{|l|}
$(0.17719)$ \\
\end{tabular} & \begin{tabular}{|l|}
$(0.14807)$ \\
\end{tabular} & \begin{tabular}{|l|}
$(0.18443)$ \\
\end{tabular} & \begin{tabular}{|l|l|l}
$(0.17568)$ \\
\end{tabular} \\
\hline \multirow[t]{2}{*}{9} & -0.335662 & -0.108606 & -0.216101 & 0.267250 & 0.102756 & -0.663036 & -0.132620 & -0.127760 \\
\hline & \begin{tabular}{|l}
$(0.28875)$ \\
\end{tabular} & \begin{tabular}{|l|}
$(0.17362)$ \\
\end{tabular} & \begin{tabular}{|l|}
$(0.23066)$ \\
\end{tabular} & \begin{tabular}{|l|}
$(0.18746)$ \\
\end{tabular} & \begin{tabular}{|l}
$(0.18171)$ \\
\end{tabular} & \begin{tabular}{|l|}
$(0.15282)$ \\
\end{tabular} & \begin{tabular}{|l}
$(0.18635)$ \\
\end{tabular} & $(0.18181)$ \\
\hline \multirow[t]{2}{*}{10} & -0.343646 & -0.144028 & -0.253893 & 0.266827 & 0.109119 & -0.677194 & -0.131746 & -0.152484 \\
\hline & \begin{tabular}{|l}
$(0.30088)$ \\
\end{tabular} & \begin{tabular}{|l}
$(0.17858)$ \\
\end{tabular} & \begin{tabular}{|l}
$(0.23820)$ \\
\end{tabular} & \begin{tabular}{|l|}
$(0.19301)$ \\
\end{tabular} & \begin{tabular}{|l|}
$(0.18777)$ \\
\end{tabular} & \begin{tabular}{|l|}
$(0.15743)$ \\
\end{tabular} & \begin{tabular}{|l|}
$(0.19178)$ \\
\end{tabular} & $\mid(0.18976)$ \\
\hline \multicolumn{9}{|c|}{ Response of INDEKS_KURS: } \\
\hline Period & BI_RATE & BOPO & FDR & IHK & IPI & KURS & INF & ROA \\
\hline \multirow[t]{2}{*}{1} & 0.169271 & 0.106602 & 0.104212 & 0.957837 & 0.014773 & 1.435948 & 0.000000 & 0.000000 \\
\hline & $(0.19179)$ & $(0.19115)$ & $(0.19080)$ & $(0.17534)$ & $(0.15858)$ & \begin{tabular}{|l}
$(0.11213)$ \\
\end{tabular} & $(0.00000)$ & $(0.00000)$ \\
\hline \multirow[t]{2}{*}{2} & -0.236733 & -0.079455 & -0.197650 & 0.911852 & 0.121207 & \begin{tabular}{|l|}
1.101709 \\
\end{tabular} & -0.332298 & -0.179832 \\
\hline & $(0.25095)$ & $(0.26023)$ & $(0.25166)$ & $(0.24668)$ & $(0.23615)$ & $\begin{array}{l}(0.21574) \\
\end{array}$ & $(0.20220)$ & $(0.20603)$ \\
\hline \multirow[t]{2}{*}{3} & -0.310685 & -0.122570 & -0.287176 & 0.489017 & 0.174343 & 0.937127 & -0.353332 & -0.138532 \\
\hline & $(0.28101)$ & $(0.28712)$ & $(0.26274)$ & $(0.24231)$ & $(0.26966)$ & \begin{tabular}{|l}
$(0.19377)$ \\
\end{tabular} & \begin{tabular}{|l}
$(0.23594)$ \\
\end{tabular} & $(0.24806)$ \\
\hline \multirow[t]{2}{*}{4} & -0.242040 & -0.201097 & -0.334181 & 0.179971 & 0.227082 & 0.884671 & -0.206494 & -0.118581 \\
\hline & $(0.28274)$ & $(0.26370)$ & $(0.27034)$ & $(0.24155)$ & $(0.26797)$ & $(0.18417)$ & $(0.24368)$ & $(0.27172)$ \\
\hline \multirow[t]{2}{*}{5} & -0.100406 & -0.119 & -0.298725 & -0.00 & 0.190407 & 0.829330 & -0.11 & -0.043219 \\
\hline & $(0.28790)$ & $(0.23167)$ & $(0.26606)$ & $(0.24232)$ & $(0.24446)$ & $(0.17703)$ & $(0.25484)$ & $(0.25628)$ \\
\hline \multirow[t]{2}{*}{6} & 0.021809 & -0.062086 & -0.175620 & -0.137486 & 0.086923 & \begin{tabular}{|l|}
0.852952 \\
\end{tabular} & -0.045441 & 0.037483 \\
\hline & \begin{tabular}{|l}
$(0.29419)$ \\
\end{tabular} & \begin{tabular}{|l}
$(0.20873)$ \\
\end{tabular} & \begin{tabular}{|l}
$(0.26620)$ \\
\end{tabular} & \begin{tabular}{|l|}
$(0.23748)$ \\
\end{tabular} & \begin{tabular}{|l}
$(0.23163)$ \\
\end{tabular} & \begin{tabular}{|l|}
$(0.17418)$ \\
\end{tabular} & \begin{tabular}{|l}
$(0.25134)$ \\
\end{tabular} & $(0.23182)$ \\
\hline \multirow[t]{2}{*}{7} & 0.089817 & -0.022237 & -0.047748 & -0.204254 & 0.025637 & \begin{tabular}{|l|}
0.885906 \\
\end{tabular} & \begin{tabular}{|l}
0.011330 \\
\end{tabular} & 0.076331 \\
\hline & \begin{tabular}{|l}
$(0.30450)$ \\
\end{tabular} & \begin{tabular}{|l}
$(0.19653)$ \\
\end{tabular} & \begin{tabular}{|l|}
$(0.26947)$ \\
\end{tabular} & \begin{tabular}{|l|}
$(0.23316)$ \\
\end{tabular} & \begin{tabular}{|l}
$(0.22509)$ \\
\end{tabular} & \begin{tabular}{|l|}
$(0.17567)$ \\
\end{tabular} & \begin{tabular}{|l}
$(0.23993)$ \\
\end{tabular} & $\mid(0.21639)$ \\
\hline \multirow[t]{2}{*}{8} & \begin{tabular}{|l|}
0.147131 \\
\end{tabular} & \begin{tabular}{|l|}
0.036177 \\
\end{tabular} & \begin{tabular}{|l|}
0.051161 \\
\end{tabular} & -0.244494 & -0.015904 & \begin{tabular}{|l|}
0.912169 \\
\end{tabular} & \begin{tabular}{|l|}
0.041507 \\
\end{tabular} & 0.114464 \\
\hline & \begin{tabular}{|l}
$(0.31980)$ \\
\end{tabular} & \begin{tabular}{|l}
$(0.19916)$ \\
\end{tabular} & \begin{tabular}{|l|}
$(0.27493)$ \\
\end{tabular} & \begin{tabular}{|l|}
$(0.23170)$ \\
\end{tabular} & \begin{tabular}{|l}
$(0.22293)$ \\
\end{tabular} & \begin{tabular}{|l|}
$(0.18044)$ \\
\end{tabular} & \begin{tabular}{|l|}
$(0.23137)$ \\
\end{tabular} & $(0.21240)$ \\
\hline 9 & \begin{tabular}{|l|}
0.191959 \\
\end{tabular} & \begin{tabular}{|l|}
0.083090 \\
\end{tabular} & 0.137326 & -0.281476 & \begin{tabular}{|c|}
-0.049790 \\
\end{tabular} & \begin{tabular}{|l|}
0.939105 \\
\end{tabular} & \begin{tabular}{|l|}
0.064811 \\
\end{tabular} & \begin{tabular}{|l|l|}
0.157392 \\
\end{tabular} \\
\hline & \begin{tabular}{|l|}
$(0.33754)$ \\
\end{tabular} & \begin{tabular}{|l}
$(0.20840)$ \\
\end{tabular} & \begin{tabular}{|l}
$(0.28320)$ \\
\end{tabular} & \begin{tabular}{|l|}
$(0.23393)$ \\
\end{tabular} & \begin{tabular}{|l|}
$(0.22589)$ \\
\end{tabular} & \begin{tabular}{|l|}
$(0.18633)$ \\
\end{tabular} & \begin{tabular}{|l|}
$(0.22867)$ \\
\end{tabular} & $(0.21746)$ \\
\hline 10 & 0.224638 & 0.118616 & 0.210166 & -0.311895 & -0.070631 & 0.960253 & 0.089416 & 0.190552 \\
\hline & $(0.35655)$ & $(0.21892)$ & $(0.29294)$ & $(0.24006)$ & $(0.23189)$ & $(0.19396)$ & $(0.23222)$ & $(0.22735)$ \\
\hline Respc & se of INF: & & & & & & & \\
\hline Period & BI_RATE & BOPO & FDR & IHK & IPI & KURS & INF & ROA \\
\hline 1 & 0.284428 & -0.027014 & \begin{tabular}{|l|}
0.097098 \\
\end{tabular} & 0.122121 & -0.081297 & \begin{tabular}{|l|}
-0.023983 \\
\end{tabular} & \begin{tabular}{|l|}
0.626306 \\
\end{tabular} & 0.000000 \\
\hline & \begin{tabular}{|l}
$(0.07530)$ \\
\end{tabular} & \begin{tabular}{|l|}
$(0.07192)$ \\
\end{tabular} & \begin{tabular}{|l}
$(0.07149)$ \\
\end{tabular} & $\begin{array}{l}(0.07044) \\
\end{array}$ & \begin{tabular}{|l}
$(0.06951)$ \\
\end{tabular} & \begin{tabular}{|l}
$(0.06919)$ \\
\end{tabular} & \begin{tabular}{|l}
$(0.04891)$ \\
\end{tabular} & $(0.00000)$ \\
\hline
\end{tabular}

ISSN 2774-3918 (online), https://ksshr.kresnanusantara.co.id. Published by Kresna Nusantara

Copyright (c) Author(s). This is an open-access article distributed under the terms of the Creative Commons Attribution License (CC BY). To view a copy of this license, visit https://creativecommons.org/licenses/by/4.0/. 


\section{Kresna Social Science and Humanities Research}

Proceedings of the International Conference On Ummah:

Digital Innovation, Humanities And Economy (ICU: DIHEc) 2020

https://doi.10.30874/ksshr.63

\begin{tabular}{|c|c|c|c|c|c|c|c|c|}
\hline 2 & 0.470246 & 0.189009 & 0.071099 & 0.132030 & -0.149033 & -0.014980 & 0.528466 & 0.003799 \\
\hline & $(0.10776)$ & \begin{tabular}{|l|}
$(0.10440)$ \\
\end{tabular} & $(0.09983)$ & $(0.10149)$ & $(0.10035)$ & \begin{tabular}{|l}
$(0.09821)$ \\
\end{tabular} & \begin{tabular}{|l|}
$(0.09134)$ \\
\end{tabular} & $(0.08395)$ \\
\hline \multirow[t]{2}{*}{3} & 0.400216 & \begin{tabular}{|l|}
0.147708 \\
\end{tabular} & 0.193672 & 0.114343 & -0.234011 & \begin{tabular}{|l|}
0.052750 \\
\end{tabular} & \begin{tabular}{|l|}
0.384571 \\
\end{tabular} & -0.011937 \\
\hline & \begin{tabular}{|l|}
$(0.13269)$ \\
\end{tabular} & \begin{tabular}{|l|}
$(0.12953)$ \\
\end{tabular} & $(0.11878)$ & $(0.11184)$ & \begin{tabular}{|l}
$(0.12103)$ \\
\end{tabular} & \begin{tabular}{|l}
$(0.09483)$ \\
\end{tabular} & \begin{tabular}{|l|}
$(0.11362)$ \\
\end{tabular} & $(0.11394)$ \\
\hline \multirow[t]{2}{*}{4} & 0.249118 & 0.050291 & 0.197514 & 0.143194 & -0.178430 & \begin{tabular}{|l}
0.056831 \\
\end{tabular} & 0.277654 & -0.103803 \\
\hline & \begin{tabular}{|l}
$(0.13805)$ \\
\end{tabular} & \begin{tabular}{|l|}
$(0.12850)$ \\
\end{tabular} & $(0.12944)$ & $(0.11484)$ & \begin{tabular}{|l}
$(0.12806)$ \\
\end{tabular} & \begin{tabular}{|l}
$(0.09111)$ \\
\end{tabular} & \begin{tabular}{|l|}
$(0.12201)$ \\
\end{tabular} & $(0.13272)$ \\
\hline \multirow[t]{2}{*}{5} & 0.160400 & \begin{tabular}{|l|}
0.050875 \\
\end{tabular} & 0.127601 & 0.145679 & -0.114903 & \begin{tabular}{|l|}
0.016332 \\
\end{tabular} & \begin{tabular}{|l|}
0.169611 \\
\end{tabular} & -0.101518 \\
\hline & \begin{tabular}{|l}
$(0.13535)$ \\
\end{tabular} & \begin{tabular}{|l}
$(0.10968)$ \\
\end{tabular} & $(0.12568)$ & $(0.11249)$ & $(0.11552)$ & \begin{tabular}{|l}
$(0.08231)$ \\
\end{tabular} & \begin{tabular}{|l}
$(0.12504)$ \\
\end{tabular} & $(0.12796)$ \\
\hline \multirow[t]{2}{*}{6} & 0.094090 & 0.030469 & 0.074883 & 0.111694 & -0.081529 & -0.000608 & 0.108405 & -0.063888 \\
\hline & $(0.13102)$ & \begin{tabular}{|l}
$(0.08639)$ \\
\end{tabular} & $(0.11791)$ & $(0.10364)$ & $(0.09842)$ & \begin{tabular}{|l}
$(0.07403)$ \\
\end{tabular} & $(0.11952)$ & $(0.10926)$ \\
\hline \multirow[t]{2}{*}{7} & 0.033609 & \begin{tabular}{|l|}
0.010769 \\
\end{tabular} & 0.034939 & 0.088071 & -0.043658 & \begin{tabular}{|l|}
-0.018736 \\
\end{tabular} & \begin{tabular}{|l|}
0.081897 \\
\end{tabular} & -0.050731 \\
\hline & \begin{tabular}{|l|}
$(0.12756)$ \\
\end{tabular} & \begin{tabular}{|l|}
$(0.06669)$ \\
\end{tabular} & $(0.10953)$ & $(0.09122)$ & \begin{tabular}{|l|}
$(0.08272)$ \\
\end{tabular} & \begin{tabular}{|l}
$(0.06697)$ \\
\end{tabular} & \begin{tabular}{|l|}
$(0.10567)$ \\
\end{tabular} & $(0.08961)$ \\
\hline \multirow[t]{2}{*}{8} & -0.003303 & 0.009835 & 0.004054 & 0.073349 & -0.022558 & -0.034248 & 0.057082 & -0.041193 \\
\hline & \begin{tabular}{|l|}
$(0.12520)$ \\
\end{tabular} & \begin{tabular}{|l|}
$(0.05434)$ \\
\end{tabular} & $(0.10018)$ & $(0.07773)$ & \begin{tabular}{|l}
$(0.06800)$ \\
\end{tabular} & \begin{tabular}{|l}
$(0.06092)$ \\
\end{tabular} & \begin{tabular}{|l|}
$(0.08830)$ \\
\end{tabular} & $(0.07383)$ \\
\hline \multirow[t]{2}{*}{9} & -0.030013 & 0.003477 & -0.011786 & 0.060703 & -0.014710 & -0.041154 & 0.036062 & -0.032063 \\
\hline & $(0.12302)$ & \begin{tabular}{|l|}
$(0.04785)$ \\
\end{tabular} & $(0.09022)$ & $(0.06454)$ & $(0.05488)$ & \begin{tabular}{|l}
$(0.05492)$ \\
\end{tabular} & \begin{tabular}{|l|}
$(0.07114)$ \\
\end{tabular} & $(0.06262)$ \\
\hline \multirow[t]{2}{*}{10} & -0.051357 & -0.005439 & -0.022038 & 0.052258 & -0.006832 & -0.045941 & 0.020202 & -0.028388 \\
\hline & \begin{tabular}{|l|}
$(0.12029)$ \\
\end{tabular} & $(0.04340)$ & $(0.07992)$ & $(0.05291)$ & $(0.04376)$ & $(0.04927)$ & $(0.05646)$ & $(0.05492)$ \\
\hline \multicolumn{9}{|c|}{ Response of ROA: } \\
\hline Period & BI_RATE & BOPO & FDR & IHK & IPI & KURS & INF & ROA \\
\hline \multirow[t]{2}{*}{1} & -0.040593 & -0.057269 & 0.126238 & 0.003614 & \begin{tabular}{|l|}
0.039274 \\
\end{tabular} & \begin{tabular}{|l|}
0.017835 \\
\end{tabular} & -0.016790 & 0.277531 \\
\hline & $(0.03478)$ & \begin{tabular}{|l|}
$(0.03435)$ \\
\end{tabular} & $(0.03260)$ & $(0.03107)$ & $(0.03092)$ & $(0.03074)$ & \begin{tabular}{|l|}
$(0.03068)$ \\
\end{tabular} & $(0.02167)$ \\
\hline \multirow[t]{2}{*}{2} & -0.076890 & 0.015017 & 0.128796 & 0.022440 & 0.030081 & 0.031682 & -0.004382 & 0.150657 \\
\hline & $(0.04135)$ & $(0.04292)$ & $(0.04005)$ & $(0.03991)$ & $(0.03983)$ & \begin{tabular}{|l}
$(0.03896)$ \\
\end{tabular} & \begin{tabular}{|l|l}
$(0.03989)$ \\
\end{tabular} & $(0.03916)$ \\
\hline \multirow[t]{2}{*}{3} & -0.081982 & 0.093626 & 0.109121 & 0.041740 & 0.038176 & 0.042723 & -0.007000 & 0.107496 \\
\hline & $(0.04555)$ & \begin{tabular}{|l|}
$(0.04688)$ \\
\end{tabular} & $(0.04087)$ & $(0.03604)$ & \begin{tabular}{|l|}
$(0.04249)$ \\
\end{tabular} & \begin{tabular}{|l|}
$(0.02944)$ \\
\end{tabular} & \begin{tabular}{|l|}
$(0.03903)$ \\
\end{tabular} & $(0.04130)$ \\
\hline \multirow[t]{2}{*}{4} & -0.091604 & \begin{tabular}{|l|}
0.063156 \\
\end{tabular} & 0.082289 & 0.033384 & \begin{tabular}{|l|}
0.017465 \\
\end{tabular} & \begin{tabular}{|l|}
0.044865 \\
\end{tabular} & \begin{tabular}{|c|}
-0.014207 \\
\end{tabular} & 0.071786 \\
\hline & $(0.04516)$ & $(0.04098)$ & $(0.04205)$ & $(0.03735)$ & \begin{tabular}{|l}
$(0.04099)$ \\
\end{tabular} & \begin{tabular}{|l}
$(0.03001)$ \\
\end{tabular} & $(0.04084)$ & $(0.04468)$ \\
\hline \multirow[t]{2}{*}{5} & -0.101648 & \begin{tabular}{|l|}
0.038049 \\
\end{tabular} & 0.060498 & 0.023063 & \begin{tabular}{|l|}
0.019591 \\
\end{tabular} & \begin{tabular}{|l|}
0.020948 \\
\end{tabular} & \begin{tabular}{|l|}
-0.014911 \\
\end{tabular} & 0.046521 \\
\hline & $(0.04551)$ & \begin{tabular}{|l|}
$(0.03549)$ \\
\end{tabular} & $(0.04069)$ & $(0.03631)$ & \begin{tabular}{|l}
$(0.03694)$ \\
\end{tabular} & \begin{tabular}{|l}
$(0.02667)$ \\
\end{tabular} & \begin{tabular}{|l|}
$(0.04137)$ \\
\end{tabular} & $(0.04115)$ \\
\hline \multirow[t]{2}{*}{6} & -0.091217 & 0.026752 & 0.039496 & 0.009472 & 0.016324 & 0.006689 & -0.016023 & 0.038660 \\
\hline & $(0.04516)$ & \begin{tabular}{|l|}
$(0.02999)$ \\
\end{tabular} & $(0.03980)$ & $(0.03468)$ & \begin{tabular}{|l}
$(0.03379)$ \\
\end{tabular} & \begin{tabular}{|l|}
$(0.02581)$ \\
\end{tabular} & \begin{tabular}{|l|}
$(0.03993)$ \\
\end{tabular} & $(0.03703)$ \\
\hline \multirow[t]{2}{*}{7} & -0.079598 & 0.019734 & 0.028625 & -0.001098 & 0.013292 & -0.000429 & -0.012773 & 0.037698 \\
\hline & $(0.04523)$ & \begin{tabular}{|l}
$(0.02540)$ \\
\end{tabular} & $(0.03854)$ & $(0.03229)$ & \begin{tabular}{|l}
$(0.03054)$ \\
\end{tabular} & \begin{tabular}{|l}
$(0.02432)$ \\
\end{tabular} & \begin{tabular}{|l}
$(0.03689)$ \\
\end{tabular} & $(0.03274)$ \\
\hline \multirow[t]{2}{*}{8} & -0.069115 & 0.016230 & 0.022075 & -0.006050 & 0.010767 & -0.003524 & -0.009334 & 0.034417 \\
\hline & \begin{tabular}{|l|}
$(0.04542)$ \\
\end{tabular} & \begin{tabular}{|l|}
$(0.02257)$ \\
\end{tabular} & $(0.03701)$ & $(0.02950)$ & \begin{tabular}{|l}
$(0.02727)$ \\
\end{tabular} & \begin{tabular}{|l|}
$(0.02328)$ \\
\end{tabular} & \begin{tabular}{|l|}
$(0.03312)$ \\
\end{tabular} & $(0.02930)$ \\
\hline \multirow[t]{2}{*}{9} & -0.059954 & \begin{tabular}{|l|}
0.015291 \\
\end{tabular} & 0.018332 & -0.007617 & \begin{tabular}{|l}
0.008095 \\
\end{tabular} & \begin{tabular}{|l|}
-0.004725 \\
\end{tabular} & -0.007863 & 0.031012 \\
\hline & $(0.04556)$ & \begin{tabular}{|l|}
$(0.02068)$ \\
\end{tabular} & $(0.03536)$ & \begin{tabular}{|l}
$(0.02680)$ \\
\end{tabular} & $(0.02431)$ & \begin{tabular}{|l|}
$(0.02212)$ \\
\end{tabular} & \begin{tabular}{|l}
$(0.02951)$ \\
\end{tabular} & $(0.02679)$ \\
\hline \multirow[t]{2}{*}{10} & -0.052599 & 0.013447 & 0.016025 & -0.007907 & 0.005732 & -0.004648 & -0.007357 & 0.027404 \\
\hline & \begin{tabular}{|l|}
$(0.04551)$ \\
\end{tabular} & \begin{tabular}{|l|}
$(0.01913)$ \\
\end{tabular} & $(0.03345)$ & $(0.02430)$ & \begin{tabular}{|l|}
$(0.02152)$ \\
\end{tabular} & \begin{tabular}{|l}
$(0.02099)$ \\
\end{tabular} & \begin{tabular}{|l}
$(0.02625)$ \\
\end{tabular} & $(0.02482)$ \\
\hline
\end{tabular}

Source : Proceed author with software

This impulse response function (IRF) will provide information about the magnitude and how long the proportion of shock a variable to the variable itself and then see the amount of shock proportion of other variables to the variable. Impulse response (ROA) in the first period, ROA is strongly influenced by FDR shock (12.6\%) while the period of the period of shock ROA and other variables still have no effect. From the 1st to the 10th period, the proportion of ROA shock to other variables increases, and the influence of the ROA variable itself decreases to the 10th period. Thus the largest

ISSN 2774-3918 (online), https://ksshr.kresnanusantara.co.id. Published by Kresna Nusantara

Copyright (C) Author(s). This is an open-access article distributed under the terms of the Creative Commons Attribution License (CC BY).

To view a copy of this license, visit https://creativecommons.org/licenses/by/4.0/. 


\section{Kresna Social Science and Humanities Research}

Proceedings of the International Conference On Ummah:

Digital Innovation, Humanities And Economy (ICU: DIHEc) 2020

https://doi.10.30874/ksshr.63

contribution is contributed by the FDR variable that affects the ROA, followed by the variable IPI, so next.

\section{Forecast error variance decomposition (FEVD)}

As for the next test using forecast error variance decomposition (FEVD). This FEVD is a test for data analysis. If you want to know the response of a particular variable as measured by the variation of the variance error error in that variable caused by the shock of other variables in the VAR equation system, the Forecast Error Variance Decomposition FEVD analysis method will be used. Variance decomposition can provide information on the contribution variability of each innovation randomly to the variables in the VAR system in [10]. This method of analysis provides an overview of the dynamics of variables in the system of VAR equations. The following test results for FEVD in this study, as presented in Figure 1.

Figure 1 : Result for forecast error variance decomposition (FEVD) 


\section{Kresna Social Science and Humanities Research}

Proceedings of the International Conference On Ummah:

Digital Innovation, Humanities And Economy (ICU: DIHEc) 2020 https://doi.org/10.30874/ksshr.4

ISSN 2774-3918 (online), https://ksshr.kresnanusantara.co.id. Published by Kresna Nusantara

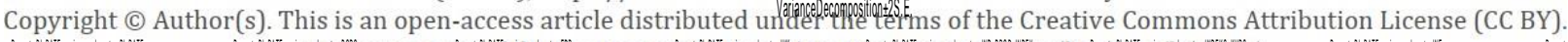

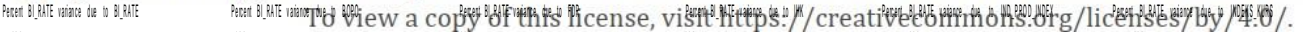
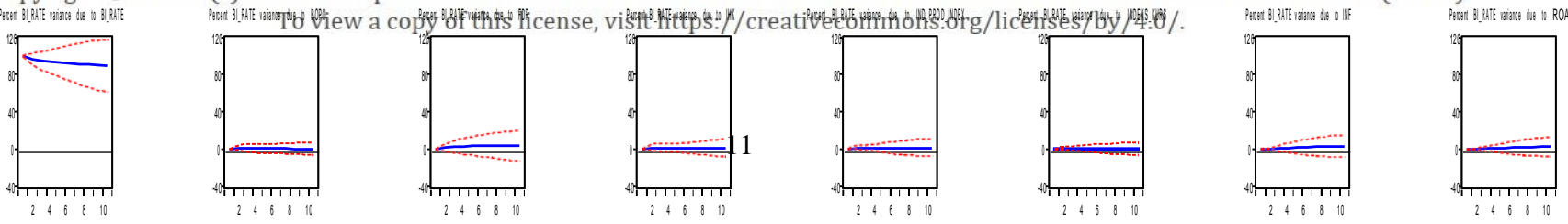

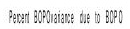

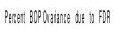

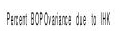

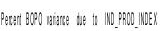

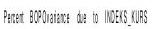




\section{Kresna Social Science and Humanities Research}

Proceedings of the International Conference On Ummah:

Digital Innovation, Humanities And Economy (ICU: DIHEc) 2020

https://doi.org/10.30874/ksshr.4

\section{Source : Proceed author with software}

Modeling FEVD, beginning with modeling VAR. VAR model is quite difficult to interpret and complicated enough for reasoning so that we will more easily do the analysis by looking at the proposed IRF VAR model output. This IRF we need to know how the effect of shock in the economy. IRF describes how the rate of shock of a variable against other variables so through this IRF, can know the influence of the occurrence of a shock / shock of a variable against other variables. More greatly, we will know how long the shock effect will be lost so that the equilibrium / economic equilibrium point recovers as before economic shocks such as the monetary crisis. Seen in figure 1 above the shock rate and shock of a variable seen initially approaching the equilibrium point, after which, it is long to stay away from the equilibrium point for the entire variable. At the beginning of the period ie the first month until the 10th month, the macro economic variable response is still very volatile that is responding positively and negatively (up and down) since the shock or shock to the banking sector variables. Next, from the 9th to the 10th month the fluctuations begin to shrink meaning that the macroeconomic variables are no longer very volatile like the previous period. It will take about 3 years for macroeconomic variables to get back to the point of balance.

\section{CONCLUSION}

For the VAR system the most important functions in the estimation are IRF and FEVD results, since the tools to answer the research objectives of the tool used will focus on the IRF results and FEVD results. In [11] explaining that there are benefits to the use of IRF and FEVD, one of them is to show how the response of a variable from a shock on the external variable itself and other internal variables. In identifying macroeconomic variable responses and financial ratios of Islamic banking using the simultaneous model of IRF and FEVD VAR in Indonesia, it aims to produce impulse responses that are crucially dependent on the ordering of variables in the system.

\section{Reference}

1).http://bisnis.liputan6.com/read/585650/3-dampak-keberadaan-bank-syariah-bagi-makroekonomi-ri.

2).Festić, M and Bekő, J, (2008), The Banking Sector and Macroeconomic Indicators: Some Evidence for Hungary and Poland, NG, št. 5-6, Strokovni članki - Professi onal papers.

3).Quagliariello, M. (2003), "Macroeconomics indicators useful in predicting bank loan quality: evidence from Italy", Rome, Bank of Italy, paper series, no. 1. 


\section{Kresna Social Science and Humanities Research}

Proceedings of the International Conference On Ummah:

Digital Innovation, Humanities And Economy (ICU: DIHEc) 2020

https://doi.org/10.30874/ksshr.4

4).İslamoglu, M, (2015), 'The Effect of Macroeconomic Variables on Non-performing Loan Ratio of Publicly Traded Banks in Turkey", WSEAS TRANSACTIONS on BUSINESS and ECONOMICS, Volume 12.

5).Louzis, et, all., Macroeconomic and Bank Specific Determinants of Nonperforming Loans in Greece: A Comparative Study of Mortgage, Business and Consumer Loan Portfolios, Journal of Banking and Finance, Vol.36 (4), 2011.

6).ÇELIK, R. et, all. (2016), The Impacts of Changes in Macro-Economic Data on Net Working Capital: The Case of Turkey's Industrial Sector, Procedia Economics and Finance 38, 122-134, doi: 10.1016/S2212-5671(16)30184-8.

7). Saksonova, S,S. and Koḷeda, O. (2017) Evaluating the Interrelationship between Actions of Latvian Commercial Banks and Latvian Economic Growth, Procedia Engineering 178123 - 130, doi: 10.1016/j.proeng.2017.01.075.

8).Jiménez,G. and J. Saurina (2004): “Collateral, Type of Lender and Relationship Banking as Determinants of Credit Risk". Journal of Banking and Finance.

9). Reinhart, C,M. and Rogoff, K.S.(May 2010), “Growth in a Time of Debt,"American Economic Review,

Vol. 100 No. 2, 573-578.

10). Wooldridge, Jeffrey (2013). Intr3oductory Econometrics, A modern approach. South-Western, Cengage learning. ISBN 978-1-111-53104-1.

11).Barbic, T., and Jurkic, I.C., (2011), Relationship Between Macroeconomic Fundamentals and Stock Market Indices in Selected CEE Countries, EKONOMSKI PREGLED, 62(3-4) 113-133. 